\title{
DIVISION III / COMMISSION 15 / WORKING GROUP PHYSICAL STUDY OF MINOR PLANETS
}

\author{
CHAIR \\ VICE-CHAIR \\ PAST CHAIR \\ MEMBERS
}

\author{
Walter F. Huebner \\ Alberto Cellino \\ Edward F. Tedesco \\ Dominique Bockelee-Morvan, Yuehua Ma, \\ Petrus M. M. Jenniskens, Dmitrij F. Lupishko \\ Harold J. Reitsema, Gonzalo Tancredi, \\ Rita M. Shulz
}

\section{PROCEEDINGS BUSINESS MEETING on 24 August 2006}

The meeting of the Physical Properties of the Minor Planets Working Group of IAU Commission 15 took place on 24 August, and was devoted to purely scientific matters, since other topics (organization of the Minor Planet WG, need of a new web page, election of the new chairman) had been already discussed during the business meeting of Commission 15, on 22 August. A brief summary of the talks given during the meeting is given in what follows.

\section{Introduction}

The former chairman of the Minor Planet Working Group, A. Cellino, made a very short introduction, to introduce the main subjects of the discussion. The meeting was divided into two major sections, whose titles were "Asteroid Radiometry and Polarimetry" and "The Renaissance of Polarimetry", respectively. As the titles clearly show, the two parts were intimately correlated, and they were chosen because they deal with some delicate issues of the current state of the art in asteroid science, which deserve careful attention and, very likely, some possible actions by IAU Commission 15 in the next triennium.

\section{Asteroid radiometry and polarimetry}

E. Tedesco gave two consecutive talks, devoted to two complementary subjects. The first talk had the title "Issues with the Radiometric Method for Small Asteroids" and was a summary of the state of the art in the field of thermal radiometry observations of asteroids, with particular emphasis on what has been learned by the most recent observing campaigns devoted to small objects, both in the Main Belt and among the near-Earth population. In particular, the presentation emphasized the contrast between the fairly good performances of thermal radiometry as far as the determination of sizes for objects larger than $20 \mathrm{~km}$ is concerned, and the much more uncertain results concerning the determination of sizes and, even more, albedos, for objects smaller than the above value. In particular, it was emphasized that the large error bars in the determination of the albedos for small asteroids observed by means of thermal radiometry (up to $60 \%$ for objects having only one single thermal radiometry measurement) come from two main sources: the uncertainty in the thermal models to be used, and the large uncertainties in the knowledge of the absolute magnitudes of the objects.

The second talk just focused on the problem of the large errors in the absolute magnitudes (the $\mathrm{H}$ values, following the H,G system recommended by the IAU) as listed by the official sources, like the Minor Planet Center. These errors turn out to be increasingly important for objects of increasing faintness. Different investigations carried out in recent years by different authors have led to conclude that the listed values of $\mathrm{H}$ are affected by both random and systematic errors, 
which are as large as 0.5 magnitudes for objects with an estimated $\mathrm{H}$ between 14 and 15 . These large uncertainties on the absolute magnitudes have very negative effect on the performances of thermal radiometry in the determination of the albedos, and of polarimetry in the determination of the sizes (see below).

\title{
3. The renaissance of polarimetry
}

A. Cellino gave a talk summarizing the current state of the art in the field of asteroid polarimetry. Recent years have seen a substantial blossoming of polarimetric studies, taking profit of the availability of new polarimeters, large telescopes and theoretical and laboratory advances. Some examples are given by the extensive observing campaigns carried out at the CASLEO observatory, that led, among other interesting results, to the discovery of objects exhibiting previously unknown properties like (234) Barbara, and the determination of the average albedo of (25143) Itokawa before the rendez-vous with the Hayabusa space probe. After a presentation about "A polarimetry-based asteroid taxonomy", prepared by A. C. Levasseur-Regourd and given by A. Cellino (due to the fact that the author could not be present at the meeting) E. F. Tedesco gave a talk focusing about the problem of the calibration of the so-called polarimetric slope versus albedo relation, which is a hot topic because in the current phase of renaissance of polarimetry different authors are still using different calibrations, leading to unnecessary confusion.

\section{Conclusions}

Due to the fact that both the problems of the systematic errors in the $H$ absolute magnitude and the calibration of the polarimetric slope - albedo relation are important and deserve careful analysis and quick actions, it was decided, as also suggested by the new President of IAU Division III, E. Bowell, to set up urgently two Task Groups to deal with these two issues. A. Cellino took the commitment to prepare two short documents to be submitted to the President of Commission 15, in order to start the actions needed to establish these two Task Groups.

\author{
Alberto Cellino \\ former chair of the Working Group
}

